\title{
Two-dimensional electronic spectroscopy reveals ultrafast dynamics at a conical intersection in an organic photovoltaic material
}

\author{
Ephraim Sommer ${ }^{1}$, Antonietta De Sio $^{1,{ }^{*}}$ Elena Mena-Osteritz ${ }^{2}$, Peter Bäuerle ${ }^{2}$, and \\ Christoph Lienau ${ }^{1}$ \\ ${ }^{1}$ Institut für Physik, Carl von Ossietzky Universität, 26129 Oldenburg, Germany \\ ${ }^{2}$ Institut für Organische Chemie II und Neue Materialien, Universität Ulm, 89081 Ulm, Germany
}

\begin{abstract}
Two-dimensional electronic spectroscopy with sub-10-fs time resolution reveals signatures of vibronic coupling and wavepacket motion through a conical intersection in the initial charge separation dynamics of an acceptor-donor-acceptor oligomer thin film for organic solar cells.
\end{abstract}

\section{Introduction}

Photoinduced charge transfer from the donor (D) to the acceptor (A) is fundamental for the energy conversion in organic photovoltaics (OPV). Efficient charge separation on ultrafast $(<1 \mathrm{ps})$ timescales has been reported in many OPV materials. Yet at present, the microscopic mechanisms underlying this process, in particular the role of coupled electronic and nuclear motion, are controversially debated. OPV materials are complex molecular systems which may present many vibrational degrees of freedom. Quantum theory predicts that, in such large molecules, vibronic coupling to many modes may result in conical intersections (CoIns) of potential energy surfaces (PES) [1] and may thus profoundly influence the ultrafast dynamics and yield of charge transfer processes. So far, however, the importance of CoIns for the dynamics of OPV materials has not yet been discussed. Here we use two-dimensional electronic spectroscopy (2DES) with sub-10-fs time resolution to study the initial, light-induced dynamics in an A-D-A oligomer thin film which has been recently employed in highly-efficient OPV devices [2]. We show that the initial charge transfer dynamics in this material involves passage of the optically excited wavepacket through a CoIn within $<50$ fs.

\section{Results}

Thin films of a novel oligomer, based on dithieno-pyrrole as D and dicyanovinyl as A [2], are investigated with a home-built ultra-broadband 2DES spectrometer in the partially collinear pump-probe geometry. A common-path birefringent interferometer

* Corresponding author: antonietta.de.sio@uni-oldenburg.de 
is used to generate the collinear pump-pulse pair [3]. The linear absorption spectrum and the absorptive 2DES maps are shown in Fig 1.
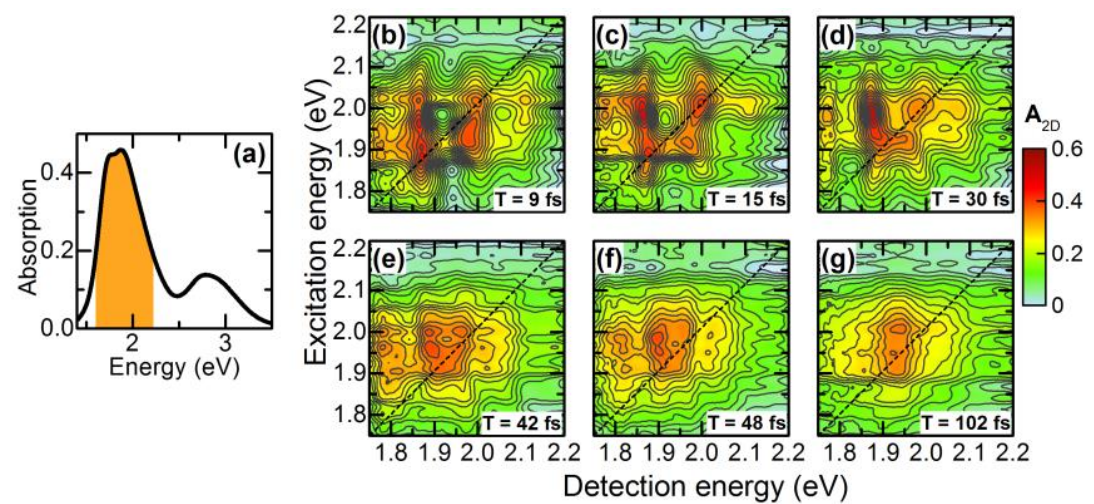

Fig. 1. Two-dimensional electronic spectroscopy (2DES) of the A-D-A oligomer thin film. (a) Linear absorption spectrum of the thin film. The excitation range is highlighted in yellow. (b-g) Absorptive 2DES maps at selected waiting times $\mathrm{T}$.

Despite the broad absorption band (Fig 1a), upon impulsive excitation of the intramolecular charge transfer (ICT) exciton at $\sim 1.9 \mathrm{eV}$, the 2DES maps at early waiting times ( $<40 \mathrm{fs}$ ) (Fig 1b) show a grid-like structure suggesting excitation of coherent vibrational wavepacket motion [4,5]. From the peak splittings, vibronic coupling to a dominant mode with oscillation period of $30 \mathrm{fs}$ can be estimated. Interestingly, the early time dynamics shows pronounced spectral peak shifts with monotonic decrease of the peak splittings, indicating that the optically excited wavepacket in the ICT state experiences a temporally rapidly varying PES. Within only $\sim 45 \mathrm{fs}$, the grid-like pattern transforms into a broader, nearly featureless peak, completely different from the structure at earlier times (Fig 1b-e). No significant spectral changes can be observed at later times (Fig $1 \mathrm{f}-\mathrm{g}$ ). Also the population dynamics of the ICT peak around $1.9 \mathrm{eV}$ shows an initial, abrupt amplitude drop together with the spectral changes at $\sim 45 \mathrm{fs}$, indicating an ultrafast depopulation of the ICT state concurrent with the disappearance of the wavepacket. At the same time, weaker oscillatory features with a distinctly different period ( $\sim 24 \mathrm{fs})$ appear and persist for the rest of the investigated timescale. All these features are difficult to reconcile with a single-mode vibronic coupling scenario, as recently reported for some polymers $[4,5]$. We argue that they are instead consistent with wavepacket motion through a CoIn.
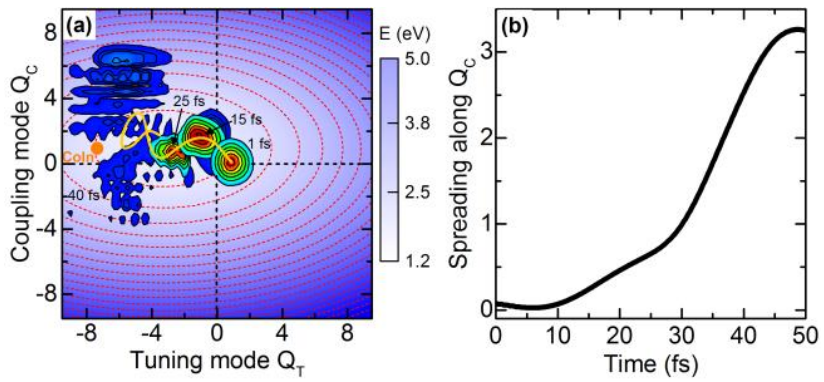

Fig. 2. Simulated wavepacket dynamics through a conical intersection based on a two-mode linear vibronic coupling model. (a) Snapshots of the simulated wavepacket dynamics projected on the 
diabatic PES of the bright state. The color gradient indicates increasing energy. The orange dot marks the position of the CoIn in the dimensionless coordinate plane. The yellow solid line shows the dynamics of the center of mass of the wavepacket. (b) Spreading of the bright state wavepacket along the coupling coordinate obtained by calculating the variance of the wavepacket distribution along the coupling coordinate.

Wavepacket simulations (Fig 2a), based on a two-modes linear vibronic coupling model describing a CoIn between an optically bright (ICT) and dark excited state, qualitatively explain several essential features of our experiments. Impulsive optical excitation launches vibrational wavepacket motion on the bright state PES starting in the Franck-Condon region. The dynamics of the center of mass (Fig 2a, yellow solid line) of the wavepacket in the vibrational coordinate plane emphasizes that the oscillation period along the coupling coordinate gradually increases as the wavepacket approaches the CoIn. This is consistent with the wavepacket moving into the anharmonic potential region induced by strong vibronic coupling close to the CoIn. Upon crossing the CoIn, at $\sim 40 \mathrm{fs}$, vibronic coupling induces rapid and efficient population decay (from the bright to the dark state) and concurrent wavepacket spreading in the PES (Fig 2a). This spreading, due to mode-mode coupling at the CoIn, can be nicely seen by computing the variance of the wavepacket distribution along the coupling coordinate (Fig 2b). Both the population transfer and the spreading cause rapid drop of the wavepacket amplitude, in agreement with the vanishing of the 2DES peak pattern in our experiments. Our 2DES results thus reveal signatures that the optically excited wavepacket in the ICT state passes a CoIn within about $45 \mathrm{fs}$.

\section{Conclusions}

Our results indicate, for the first time, that the initial steps of the light-induced charge separation dynamics in a highly efficient OPV material is governed by multimode vibronic coupling at a CoIn on a timescale of $\sim 45$ fs. Moreover, our results show the valuable insight provided by ultrafast 2DES into multimode wavepacket dynamics through a CoIn.

\section{References}

1. L.S. Cederbaum, H. Köppel, W. Domcke, Int. J. Quantum Chem. 20, 251-267, (1981).

2. D. Popovic, I. Ata, J. Krantz, S. Lucas, M. Linden, E. Mena-Osteritz, P. Bäuerle, J. Mater. Chem. C 5, 9920-9928 (2017).

3. D. Brida, C. Manzoni, G. Cerullo, Opt. Lett. 37, 3027 (2012).

4. A. De Sio, F. Troiani, M. Maiuri, J. Réhault, E. Sommer, J. Lim, S.F. Huelga, M.B. Plenio, C.A. Rozzi, G. Cerullo, E. Molinari, C. Lienau, Nat. Commun. 7, 13742 (2016).

5. A. De Sio, C. Lienau, Phys. Chem. Chem. Phys. 19, 18813-18830 (2017). 